\title{
The role of central attention in retrieval from visual short-term memory
}

\author{
Hagit Magen ${ }^{1}$
}

Published online: 28 June 2016

(C) Psychonomic Society, Inc. 2016

\begin{abstract}
The role of central attention in visual short-term memory (VSTM) encoding and maintenance is well established, yet its role in retrieval has been largely unexplored. This study examined the involvement of central attention in retrieval from VSTM using a dual-task paradigm. Participants performed a color change-detection task. Set size varied between 1 and 3 items, and the memory sample was maintained for either a short or a long delay period. A secondary tone discrimination task was introduced at the end of the delay period, shortly before the appearance of a central probe, and occupied central attention while participants were searching within VSTM representations. Similarly to numerous previous studies, reaction time increased as a function of set size reflecting the occurrence of a capacity-limited memory search. When the color targets were maintained over a short delay, memory was searched for the most part without the involvement of central attention. However, with a longer delay period, the search relied entirely on the operation of central attention. Taken together, this study demonstrates that central attention is involved in retrieval from VSTM, but the extent of its involvement depends on the duration of the delay period. Future studies will determine whether the type of memory search (parallel or serial) carried out during retrieval depends on the nature of the attentional mechanism involved the task.
\end{abstract}

Keywords VSTM $\cdot$ Retrieval $\cdot$ Central attention $\cdot$ PRP

Hagit Magen

msmagen@mail.huji.ac.il

1 School of Occupational Therapy, Faculty of Medicine of Hadassah and the Hebrew University, Jerusalem, Israel
Successful performance in the change-detection task, a widely used measure of visual short-term memory (VSTM) entails the encoding of information, maintaining it and retrieving it when necessary (Luck \& Vogel, 1997; Phillips, 1974). Numerous studies demonstrated that at least two attentional mechanisms are involved in encoding and maintenance in VSTM, namely visual-spatial attention and central attention (Allen, Baddeley, \& Hitch 2006; Gazzaley \& Nobre, 2012; Jolicoeur \& Dell'Acqua, 1998). Attention has been implicated in retrieval from VSTM as well, but the main focus has been on visual-spatial attention (Dell'Acqua, Sessa, Toffanin, Luria, \& Jolicoeur, 2010; Hyun, Woodman, Vogel, \& Hollingworth, 2009; Kuo, Rao, Lepsien, \& Nobre, 2009; Theeuwes, Kramer, \& Irwin, 2011). Given its role in encoding and maintenance, this study explored whether central attention is involved in retrieval from VSTM as well.

In the change-detection task, participants are presented with a probe at the end of a delay period and are asked whether the probe and the initial memory sample are identical or whether a change has occurred in the probe display. It is assumed that the probe triggers a search process within the maintained representations (Sternberg, 1966). One of the most robust finding in studies on short-term memory is a linear increase in reaction-time (RT) as a function of the memory sample set size, which was initially considered the result of an exhaustive serial search through the memorized items (Sternberg, 1966). However, a linear increase in RT has been observed when either serial or parallel search processes are implemented, and the type of search may depend on specific task parameters (Donkin \& Nosofsky, 2012; Sternberg, 1975).

When the probe display contains one central item (as in this study), the search process focuses on finding a match between the probe and any of the representations held in VSTM. The search focuses on finding a match because when set size is larger than one, the single probe in the match condition is 
always different from at least one of the items in memory. A growing number of studies suggest that searching within VSTM for a match requires the operation of visual-spatial attention (Dell'Acqua et al., 2010; Kuo et al., 2009). For instance, Kuo et al. (2009) tested participants in a changedetection task with a short-delay period $(800-1,300 \mathrm{~ms})$ and a single central probe. They used electroencephalography (EEG) to examine whether the N2pc, a marker of attentional shifts toward target locations during perceptual visual search (Luck \& Hillyard, 1994), is elicited when the search occurs within VSTM representations. The authors found a typical $\mathrm{N} 2 \mathrm{pc}$ response toward the original location of the memorized target that matched the probe, demonstrating that visualspatial attention shifted to that location. The N2pc response was unaffected by the memory set size, indicating that the search was largely capacity free. Dell'Aqua et al., (2010) used a similar design and also identified an N2pc-like response in VSTM search. Nevertheless in their study the N2pc was attenuated by an increase in memory set size, mirroring the effect of set size on RT.

The observations that searching within VSTM representations involves shifts of visual-spatial attention to memorized locations, lend further support to the suggestion that the spatial configuration of the memory sample is preserved in VSTM (e.g., Gratton, 1998). However, the spatial configuration of the memory sample decays over time and ceases to affect performance when the delay period exceeds 6 seconds, and the memorized representations become more abstract and are often recoded phonologically (Baddeley, 1986; Treisman \& Zhang, 2006). Thus, the involvement of visual-spatial attention in retrieval may be restricted to short-delay durations of up to several seconds. Central attention may be involved in retrieval with longer delays, given its roles in encoding and maintenance. However, central attention may also be involved in retrieval following short delays, as suggested by two-stage models of retrieval in VSTM (Kuo et al., 2009; Yin, Gao, Jin, Ding, Liang, \& Shen, 2012). These models suggest that shifts of visual-spatial attention during retrieval are followed by a second slow, capacity-limited process. Using EEG, Yin et al. identified neural markers of central executive attention that were active during this hypothesized retrieval phase.

This study explored the role of central attention in retrieval from VSTM, using the psychological refractory period (PRP) paradigm. The PRP has been used to study the role of central attention in processes such as response selection, VSTM consolidation and retrieval from long-term memory (Green, Johnston, \& Ruthruff, 2011; Jolicoeur \& Dell'Acqua, 1998; Magen \& Cohen, 2010; Pashler, 1994). In the PRP paradigm, participants make speeded responses to two tasks whose input is presented in succession, in a prespecified order. The stimulus onset asynchrony (SOA) between the stimuli of the first and the second tasks (T1 and T2, respectively) is manipulated. Typically, RT for T2 get progressively longer with shorter
SOAs, a pattern known as the PRP effect (Pashler, 1994). The postponement in $\mathrm{T} 2$ processing is considered to be the result of a limitation in a shared central attentional mechanism (i.e., executive functions) which is allocated first to T1, and therefore cannot process some aspects of $\mathrm{T} 2$ concurrently (Logan \& Gordon, 2001). Besides dual-task processing, central attention has been implicated in other domains, such as working memory, VSTM and task-switching (Baddeley, 1986; Jolicoeur \& Dell'Acqua, 1998; Logan \& Gordon, 2001).

When SOA is short, the postponement in $\mathrm{T} 2$ processing creates a "cognitive slack" in T2, which ends when central attention is released from $\mathrm{T} 1$ and shifts to T2 (see Fig. 1). The slack is utilized by the locus-of-slack method to explore the involvement of central attention in various processes in $\mathrm{T} 2$. The locus-of-slack method (Schweickert, 1980) involves the manipulation of the difficulty of a certain process in T2. The occurrence of the cognitive slack leads to two possible patterns of T2 RT (RT2) related to the difference between the easy and difficult conditions. If the manipulated process in $\mathrm{T} 2$ does not require central attention (i.e., is not postponed while central attention processes T1), an underadditive pattern emerges in which the RT difference between the easy and difficult conditions is apparent in the long SOA, but is reduced in the short SOA conditions (see Fig. 1a). In this situation, the difficulty effect is absorbed into the slack in the short SOA conditions, and is not apparent in RT2. If, however, the T2 difficulty manipulation involves central attention or beyond, T2's processing is postponed until central attention is done with the processing of T1. In this case the difference between the easy and difficult conditions is unaffected by SOA, and the interaction between SOA and $\mathrm{T} 2$ difficulty manipulation is additive (see Fig. 1b).

In the current PRP study, a tone discrimination task (i.e., T1) preceded the appearance of the probe in a color changedetection task (i.e., T2). The tone discrimination task engaged central attention for control processes such as task-set configuration, which are necessary for execution of every discrimination task including T2 (Logan \& Gordon, 2001). When the SOA between the tone and the probe was short, central attention was engaged with the tone task while participants searched within VSTM. Search was done under two difficulty conditions defined by the memory set size, which was varied between one and three items. Thus, if memory search could proceed without central attention, the RT set-size effect should be absorbed in the cognitive slack and show an underadditive interaction with the PRP effect (see Fig. 1a). However, if memory search involves central attention, the search would be postponed until central attention had finished processing $\mathrm{T} 1$, and the set-size effect would be additive with the PRP effect (see Fig. 1b). Because VSTM processes may differ as a function of delay (Phillips, 1974; Sternberg, 1975; Treisman \& Zhang, 2006), the delay length was manipulated across participants in two separate experiments. 


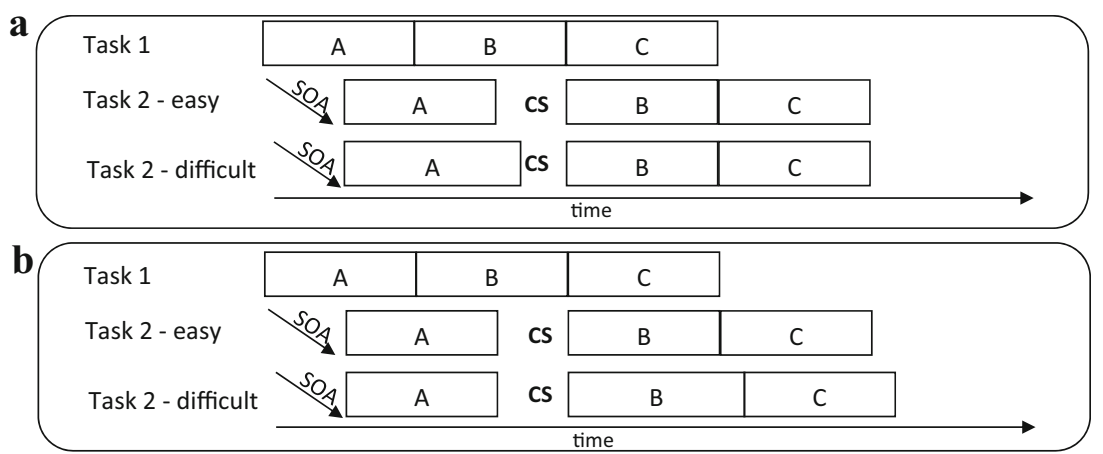

Fig. 1 Process $\mathrm{B}$ represents central attention, and processes $\mathrm{A}$ and $\mathrm{C}$ represent pre- and postcentral attention processes, respectively. CS: Cognitive slack. a: An illustration of underadditivity in the psychological refractory period (PRP) paradigm. Processes that do not require central attention proceed during the cognitive slack. As a result, the RT difference between the easy and difficult conditions is absorbed in the cognitive

\section{Method}

Participants Two groups of 18 students from The Hebrew University participated in two experiments, for course credit or payment. Each experiment consisted two separate 1-hour sessions. Participants reported having normal or corrected-tonormal vision.

Stimuli, design, and procedure Participants sat in a dimly lit room and viewed the display from a distance of $100 \mathrm{~cm}$ while resting their head on a chin rest. Each trial began with the presentation of a fixation point for $500 \mathrm{~ms}$, followed by the presentation of the memory sample for $500 \mathrm{~ms}$ (see Fig. 2). The memory sample contained one or three colored squares measuring $0.5^{\circ}$ of visual angle. The colors were chosen randomly without replacement from a set of nine colors (green, blue, red, yellow, cyan, magenta, white, brown, and purple), and appeared on a gray background in one of nine cells in a $3 \times$ 3 matrix (measuring $4.5^{0} \times 4.5^{\circ}$ of visual angle). The offset of the memory sample was followed by a delay period of 1,500 or $6,000 \mathrm{~ms}$ (manipulated across participants). At the end of the delay period (and before the appearance of the memory probe) a computer-generated tone was presented at either $300 \mathrm{~Hz}$ or $900 \mathrm{~Hz}$ for $150 \mathrm{~ms}$, requiring participants to decide whether the tone was low pitched or high pitched. The tone was followed slack. b: An illustration of additivity in the PRP paradigm. Processes that require central attention are postponed and are executed only after Task 1 has released central attention. Consequently, the difference between the two difficulty conditions of Task 2 is evident in all the stimulus onset asynchrony (SOA) conditions

by a single probe that appeared at the center of the screen until the participant responded. On $50 \%$ of the trials, the probe matched the color of one of the items in the memory sample. On the remaining trials (the change trials), the color of the probe did not match any of the items in the memory sample and was chosen randomly from the colors that did not appear on that trial. The tone and the memory probe were separated by four SOAs, each appeared on $25 \%$ of the trials. Two, were short SOAs lasting 50 and $150 \mathrm{~ms}$ with large overlap between $\mathrm{T} 1$ and $\mathrm{T} 2$ (in the $50 \mathrm{~ms}$ SOA condition, T1 and T2 stimuli overlapped). A third intermediate SOA of $300 \mathrm{~ms}$ with some overlap between the tasks, and a long SOA condition of $900 \mathrm{~ms}$ with no overlap between the tasks were used as well. Therefore, the actual delay between the memory sample and the probe varied as a function of SOA.

Participants responded with the index and middle fingers of their left hand to the high and low tones, respectively, and with the index and middle fingers of the their right hand to the match and change trials, respectively. In the short-delay experiment, each session included seven experimental blocks of 96 trials each, whereas in the long-delay experiment each session included five blocks of 64 trials each (the number of trials was adjusted to maintain a 1-h session in both experiments). Participants received at the beginning of the first session three practice blocks, 30 practice trials for the tone task

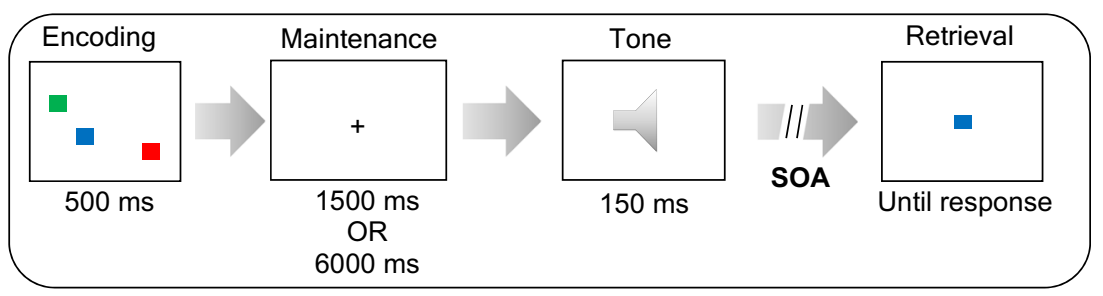

Fig. 2 An illustration of a typical trial with set-size three. In each trial participants were presented first with the memory sample, followed by a delay of $1,500 \mathrm{~ms}$ or $6,000 \mathrm{~ms}$. Following the delay, a tone discrimination

four different SOA conditions of 50, 150, 300, and $900 \mathrm{~ms}$. Note that in the $50 \mathrm{~ms} \mathrm{SOA}$ condition, T1 and T2 stimuli overlapped. (Color figure online) 
only, 12 practice trials for the VSTM task only, and 16 dualtask practice trials. Participants were asked to respond as rapidly and as accurately as possible for both tasks, and were instructed to respond first to the tone (T1) and then to the color probe (T2). An error message was presented on the screen for $500 \mathrm{~ms}$ following incorrect responses, noting whether the error was in the tone task, the memory task or both. In any case, the intertrial interval was $1,500 \mathrm{~ms}$.

\section{Results}

Similarly to other PRP experiments, data from the first session were considered practice and will not be reported. For each participant all RTs greater than 3,000 ms were first removed. Of the remaining trials, all RTs exceeding three standard deviations from the mean of each condition were removed as well (this accounted for $1.9 \%$ and $1.1 \%$ of the trials in the short- and long-delay experiments, respectively). Correct responses for $\mathrm{T} 2$ were also removed if the response to $\mathrm{T} 1$ was incorrect. Correct mean RTs and error rates for all the conditions are presented in Appendix Table 2. Statistics for all the statistical analyses are presented in Table 1.

\section{Short delay}

Tone task (T1) A significant effect of SOA was obtained with both RT and errors. RT and error rates decreased from the shortest to the longest SOA. This pattern of results is common to many PRP studies, and has no impact on the results of T2.

VSTM task (T2) The impact of SOA on RT was evaluated in a $4(\mathrm{SOA}) \times 2($ set size $) \times 2$ probe $($ match $/$ change $)$ repeatedmeasures ANOVA (see Fig. 3). The ANOVA revealed a significant effect of SOA, showing the typical PRP effect. The main effects of set size and probe and the interaction between them were also significant. Participants were slower to respond as set size increased, and overall were slower to respond in change relative to match trials. However, RT was slower in change trials only with Set Size 1, and was similar in change and match trials with Set Size 3. The probe effect did not interact significantly with SOA (see Appendix 2 for further discussion of the probe effect).

Importantly, the set-size effect was reduced in the short SOA conditions showing an underadditive interaction of set size and SOA. The three-way interaction of set size, SOA and probe was nonsignificant, showing that the underadditive interaction of set size and SOA was similar in match and change trials. However, as can be seen in Fig. 3, the set-size effect was eliminated in the two short SOA conditions in change trials, but was significant in these SOAs in match trials, demonstrating only partial underadditivity in the match condition (see Appendix 3 for statistics).

The error data revealed two significant effects of probe and set size and a significant interaction between them. Error rates were higher in match trials, but only for Set Size 3.

Table 1 Univariate inferential statistics for the short and long-delay experiments. ${ }^{\mathrm{a}} d f=(3,51) .{ }^{\mathrm{b}} d f=(1,17)$

\begin{tabular}{|c|c|c|c|c|c|c|}
\hline & & \multirow[t]{2}{*}{ Effect } & \multicolumn{2}{|l|}{ RT } & \multicolumn{2}{|c|}{ Accuracy } \\
\hline & & & $F$ & $\eta_{\mathrm{p}}^{2}$ & $F$ & $\eta_{\mathrm{p}}{ }^{2}$ \\
\hline \multirow[t]{8}{*}{ Short delay } & $\mathrm{T} 1$ & $\mathrm{SOA}$ & $6.05^{\mathrm{a}}$ & $0.262^{* * *}$ & $5.49^{\mathrm{a}}$ & $0.244^{* *}$ \\
\hline & $\mathrm{T} 2$ & SOA & $395.17^{\mathrm{a}}$ & $0.959^{* * * *}$ & $1.38^{\mathrm{a}}$ & 0.075 \\
\hline & & Set size & $43.49^{\mathrm{b}}$ & $0.719^{* * * *}$ & $46.56^{\mathrm{b}}$ & $0.733^{* * *}$ \\
\hline & & Probe & $29.35^{\mathrm{b}}$ & $0.633^{* * * *}$ & $36.26^{\mathrm{b}}$ & $0.681^{\text {***** }}$ \\
\hline & & SOA $\times$ Set size & $6.3^{\mathrm{a}}$ & $0.270^{* * *}$ & $<1^{\mathrm{a}}$ & 0.053 \\
\hline & & SOA $\times$ Probe & $1.11^{\mathrm{a}}$ & 0.061 & $<1^{\mathrm{a}}$ & 0.050 \\
\hline & & Set size $\times$ Probe & $27.26^{\mathrm{b}}$ & $0.616^{* * *}$ & $20.41^{b}$ & $0.546^{* * *}$ \\
\hline & & SOA $\times$ Set size $\times$ Probe & $<1^{\mathrm{a}}$ & 0.011 & $1.59^{\mathrm{a}}$ & 0.086 \\
\hline \multirow[t]{8}{*}{ Long delay } & $\mathrm{T} 1$ & SOA & $10.03^{\mathrm{a}}$ & $0.371^{* * *}$ & $5.86^{\mathrm{a}}$ & $0.256^{* *}$ \\
\hline & $\mathrm{T} 2$ & SOA & $319.84^{\mathrm{a}}$ & $0.950^{* * *}$ & $<1^{\mathrm{a}}$ & 0.018 \\
\hline & & Set size & $28.63^{\mathrm{b}}$ & $0.627^{* * *}$ & $26.28^{\mathrm{b}}$ & $0.607^{* * * *}$ \\
\hline & & Probe & $3.46^{\mathrm{b}}$ & 0.169 & $6.27^{\mathrm{b}}$ & $0.269^{*}$ \\
\hline & & SOA $\times$ Set size & $<1^{\mathrm{a}}$ & 0.018 & $<1^{\mathrm{a}}$ & 0.049 \\
\hline & & SOA $\times$ Probe & $<1^{\mathrm{a}}$ & 0.009 & $1.07^{\mathrm{a}}$ & 0.059 \\
\hline & & Set size $\times$ Probe & $12.44^{\mathrm{b}}$ & $0.423^{* *}$ & $7.30^{\mathrm{b}}$ & $0.301^{*}$ \\
\hline & & SOA $\times$ Set size $\times$ Probe & $<1^{\mathrm{a}}$ & 0.003 & $1.11^{\mathrm{a}}$ & 0.061 \\
\hline
\end{tabular}

$* p<.05 . * * p<.01 . * * * p<.001$

SOA: Stimulus onset asynchrony 

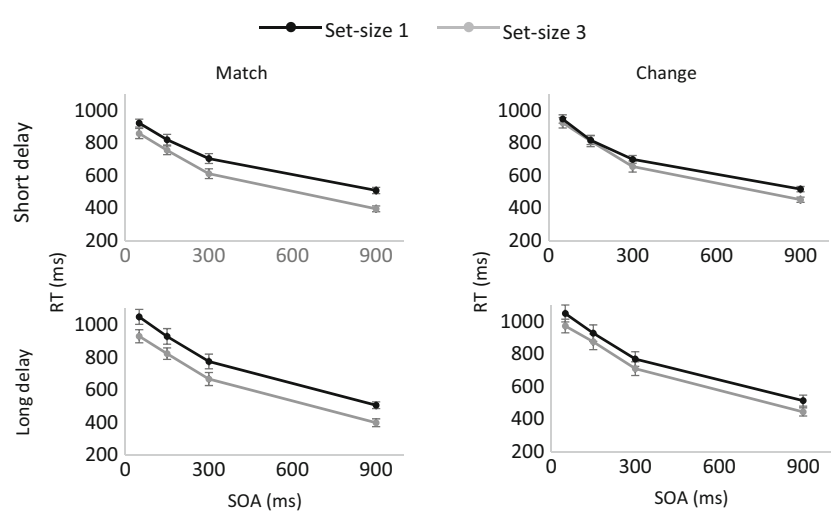

Fig. 3 RT (ms) as a function of SOA and set size, for each probe and delay conditions. Error bars represent standard error of the mean

\section{Long-delay}

Tone task (T1) The effect of SOA on RT and error rates was significant, reflecting a decrease in RT and errors from the shortest to the longest SOAs.

VSTM task (T2) The same repeated-measures ANOVA revealed significant effects of SOA and set size. The main effect of probe or its interaction with SOA were not significant. However, the probe effect interacted significantly with set size. RT was slower in change trials with Set Size 1, but was similar in the two probe conditions with Set Size 3.

Importantly, contrary to the short-delay experiment, the setsize effect was similar across the four SOA conditions, showing an additive interaction of set size and SOA. The three-way interaction of probe, set size, and SOA was nonsignificant. Thus, with the longer delay period, memory search relied on central attention in match and change trials.

The error data revealed the same pattern as in the shortdelay experiment; the effects of probe, set size, and the interaction between them were significant, reflecting higher error rates in match trials with Set Size 3.

\section{Comparing the two experiments}

The impact of the SOA manipulation on the set-size effect was compared across the two delay durations. A mixed-effects ANOVA, with the factors of SOA (50 or $900 \mathrm{~ms}$; reflecting the largest and smallest overlap between $\mathrm{T} 1$ and $\mathrm{T} 2$, respectively), probe and delay was conducted on the RT set-size effect in each of these conditions (see Fig. 4). The ANOVA revealed a significant effect of probe, $F(1,34)=16.09, p<$ $.001, \eta_{\mathrm{p}}{ }^{2}=0.321$, which did not interact with SOA or delay, $F_{\mathrm{S}}(1,34)<1$, showing that the set-size effect was significantly smaller in change trials across the SOA and delay conditions. The effects of SOA and delay were nonsignificant, $F(1,34)=$ $2.21,2.34, p \mathrm{~s}>0.14, \eta_{\mathrm{p}}{ }^{2}=0.061,0.064$, respectively. However, and most important, the interaction between SOA

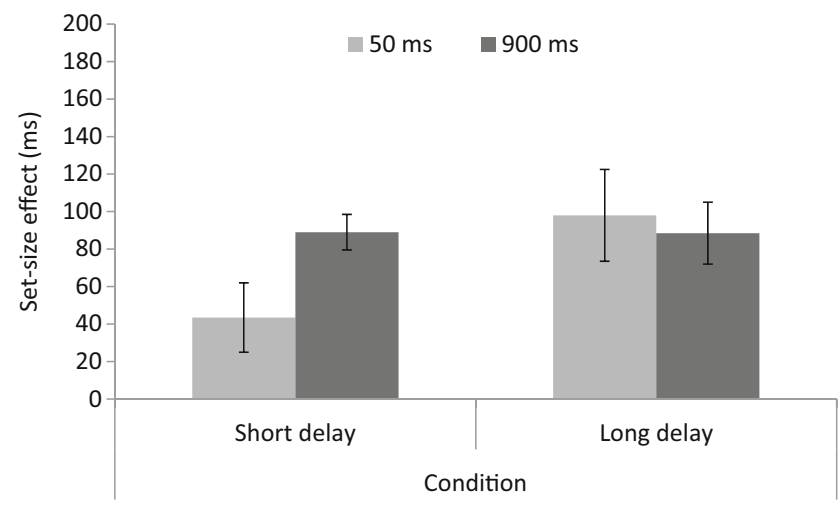

Fig. 4 The set-size effect (in ms) in the short and long SOA conditions (50 and $900 \mathrm{~ms}$, respectively) as a function of the delay period. The results are collapsed across the two probe conditions. Error bars represent standard error of the mean

and delay was significant $F(1,34)=5.22, p<.05, \eta_{\mathrm{p}}^{2}=0.133$, supporting the observations of underadditive and additive interactions of set size and SOA in the short- and long-delay conditions. The three-way interaction of SOA, probe, and delay was nonsignificant $F(1,34)<1$.

\section{Discussion}

This study used a dual-task paradigm to explore the role of central attention in retrieval from VSTM. The data replicated previous findings, showing a significant increase in RT as a function of set size, in the match and change trials. Furthermore, the results also replicated recent findings showing that the RT set-size effect was smaller in change trials (Gilchrist \& Cowan, 2014). Thus, the secondary tone task did not alter the basic memory search processes.

In the short-delay condition, the interaction of the SOA and the RT set-size effects was underadditive in the match and the change trials, demonstrating that memory search proceeded without the involvement of central attention. These results fit previous suggestions, which implicated visual-spatial attention in retrieval from VSTM (Dell'Acqua et al., 2010; Kuo et al., 2009), because studies have shown that visual-spatial attention can operate concurrently with central attention (Pashler, 1991; Reimer, Strobach, Frensch, \& Schubert, 2015). However, whereas in the short SOA conditions the set-size effect was eliminated in change trials, it was still significant in match trials. Because the set-size effect was twice as large in the match condition compared to the change condition, it is likely that it was too large to be absorbed entirely in the cognitive slack.

Alternatively, if the results indeed reflect a partial dependence of retrieval on central attention in match trials, these results could support two-stage models of retrieval, which suggest that the initial shifts of visual-spatial attention are followed by a second capacity-limited process, which involves central attention (Yin et al., 2012). This however would be restricted 
to the match trials only. Nevertheless, whereas several PRP studies demonstrated that visual-spatial and central attention can operate concurrently (e.g., Reimer et al., 2015), other studies showed that in some circumstances, visual-spatial attention depended on central attention (Dell'Acqua, Sessa, Jolicoeur, \& Robitaille, 2006). Thus, the residual set-size effect in the match condition may actually reflect visual-spatial attention processes, which require central attention, and the partial underadditivity on match trials could reflect other retrieval-related processes, which do not require central attention. Note that because the setsize effect was completely eliminated in change trials, if one assumes that memory search in match trials is partly depended on central attention, this would suggest that search is fundamentally different in match and change trials. Future studies will decide between these alternatives. Additionally, any explanation of the results should also consider the additivity of the longdelay experiment, as discussed below.

Contrary to the short-delay experiment, memory search in the match and change trials depended entirely on central attention when items were maintained for 6 seconds. One possible explanation for the additivity of the long-delay condition may be that visual-spatial attention cannot be employed to retrieve information from VSTM after 6 seconds, because the spatial configuration of the initial memory sample had dissipated (Treisman \& Zhang, 2006). Any attempt to shift visual-spatial attention to the location of the matched item may fail, and central attention is recruited to retrieve the items from VSTM. Nevertheless, additional yet unexplored factors may contribute to the difference between the two delay conditions and should be subject for future investigations considering the close links between attention and VSTM. Finally, although the RT difference between Set Sizes 1 and 3 reflects processes related to memory search, it may involve additional processes such as the need to split attention between the memorized items with Set Size 3. The additivity in the long-delay experiment demonstrates that every such potential additional process requires central attention, whereas the underadditivity in the short-delay condition shows that none of these processes rely on central attention (albeit the residual effect in the match condition discussed above).

In accordance with a recent study (Gilchrist \& Cowan, 2014), RT was slower in the change condition with Set Size 1. This probe RT effect was additive in the short- and long-delay experiments, which demonstrates that the probe effect reflects a memory process that depends on central attention. Gilchrist and Cowan suggested that the probe effect in Set Size 1 reflects a "newness check," which is slower on change trials because of proactive interference from items that participants memorized on previous trials. The current results suggest that resolving the proactive interference requires central attention.

A long-standing question in research on retrieval from STM has been whether memory search is parallel or serial. Contrary to initial suggestions, the linear increase in $\mathrm{RT}$ as a function of set size occurs with parallel and serial search processes (Donkin \& Nosofsky, 2012; Townsend, 1990). It was suggested that the type of search depends on task parameters such as set size, delay, and the memory-sample presentation durations (Donkin \& Nosofsky, 2012; Sternberg, 1975). The debate on whether retrieval is serial or parallel is still ongoing, and is beyond the scope of this study. However, this study offers new avenues for future studies that would explore the role that central attention plays is determining the type of search that is implemented during retrieval.

\section{Appendix 1}

Table 2 Mean reaction times (RTs, in ms) and percentage of errors (in parentheses) for the various stimulus onset asynchrony (SOA) conditions in the match and change trials of the two experiments

\begin{tabular}{|c|c|c|c|c|c|c|c|c|c|}
\hline \multirow[b]{3}{*}{ Experiment } & \multirow[b]{3}{*}{$\mathrm{SOA}(\mathrm{ms})$} & \multicolumn{8}{|c|}{ Probe condition } \\
\hline & & \multicolumn{4}{|l|}{ Match } & \multicolumn{4}{|l|}{ Change } \\
\hline & & 50 & 150 & 300 & 900 & 50 & 150 & 300 & 900 \\
\hline \multirow[t]{4}{*}{ Short Delay } & $\mathrm{T} 1$ & $602(3.3)$ & $581(2.4)$ & 5652.3 & $569(1.7)$ & & & & \\
\hline & $\mathrm{T} 2$ & & & & & & & & \\
\hline & Set Size 1 & 859 (2.7) & $756(3.8)$ & $612(3.1)$ & $396(5.4)$ & $925(4.2)$ & $813(4.1)$ & $656(3.8)$ & $452(1.4)$ \\
\hline & Set Size 3 & $923(18.8)$ & $821(18.2)$ & $705(16.2)$ & 509 (15.6) & 948 (10.9) & $818(11.0)$ & $700(10.6)$ & $517(9.1)$ \\
\hline \multirow[t]{4}{*}{ Long Delay } & $\mathrm{T} 1$ & $635(4.0)$ & $625(2.5)$ & $595(2.1)$ & $581(1.5)$ & & & & \\
\hline & $\mathrm{T} 2$ & & & & & & & & \\
\hline & Set Size 1 & 929 (3.9) & $822(3.4)$ & $666(3.0)$ & $397(4.3)$ & $971(4.2)$ & $874(4.3)$ & $709(2.1)$ & $443(0.8)$ \\
\hline & Set Size 3 & $1048(8.4)$ & $928(9.3)$ & $774(10.2)$ & $505(8.4)$ & 1048 (4.6) & $927(5.4)$ & $768(4.6)$ & $512(5.4)$ \\
\hline
\end{tabular}




\section{Appendix 2}

\section{Effect of probe type}

The results in this study revealed several differences in the response to the match and changed probes. These differences were unrelated to the main question of the study, because both probe conditions were underadditive in the short-delay experiment and additive in the long-delay experiment.

The results can be summarized as follows. In the shortdelay experiment, the probe effect was significant because overall participants were slower to respond in the change condition. The probe effect interacted significantly with set size because the difference between the two probes conditions was evident only with Set Size 1. Consequently, the set-size effect was smaller in the change condition. The absence of the threeway interaction between set size, SOA, and probe also shows that the smaller set-size effect in the change condition was evident across all SOA conditions.

When the error data was inspected, the set size and probe effects and the interaction between them were also significant; however, the error data showed a somewhat different pattern than RT. Participants made more errors in the match condition than the change condition, and this difference was mainly evident in Set Size 3. To verify that the RT and error data do not reflect a speed-accuracy tradeoff, follow-up $t$ tests comparing the two probe conditions were carried out separately for each set size (collapsed across the four SOAs). RT was significantly slower in the change condition with Set Size 1, $t(17)=9.90, p<.001$, and was nonsignificant with Set Size 3, $t(17)=0.68, p=.51$. The error probe effect was not significant with Set Size 1, $t(17)=0.68, p=.51$, but was significant with Set Size 3, $t(17)=5.67, p<.001$, showing higher error rates for the match condition. Thus, these data do not reflect a speed-accuracy trade-off.

The same pattern was observed in the long-delay experiment. The RT probe effect was not significant; however, it interacted significantly with the effect of set size due slower $\mathrm{RT}$ in the change condition with Set Size 1. Two additional $t$ tests verified that the probe RT difference was only significant with Set Size 1, $t(17)=2.98, p<.01$, but not with Set Size 3, $t(17)=0.32, p=.98$.

The error probe main effect was significant, as participants made more errors on match trials. The interaction of probe and set size was also significant. Here as well, the probe effect was not significant with Set Size 1, $t(17)=0.97, p=.34$, but it was significant with Set Size 3, $t(17)=2.9, p=.01$ (all tests are two-tailed).

The slower RT in the change trials with Set Size 1, and higher error rates in match trials with Set Size 3, replicate previous findings (Gilchrist \& Cowan, 2014). The results of the current study demonstrate that the same probe effects also occur with a longer delay period of 6 seconds. Furthermore, as mentioned in the Discussion section, the probe RT effect was unchanged by the SOA manipulation, which suggests that it reflects a search process that requires the operation of central attention.

\section{Appendix 3}

The set-size effect in the short-delay experiment interacted significantly with SOA, showing an underadditive interaction of set size and SOA. However, the effect was not eliminated in the match condition, whereas it was eliminated in the change condition. These impressions were verified in four $t$ tests that examined whether the set-size effect was significant in the short SOA conditions $(50 \mathrm{~ms}$ and $150 \mathrm{~ms})$ on match and change trials. The set-size effect was significant in the $50 \mathrm{~ms}$ and $150 \mathrm{~ms}$ SOAs in the match condition, $t(17)=3.14,3.45$, $p \mathrm{~s}<0.01$, respectively, but was nonsignificant in the $50 \mathrm{~ms}$ and $150 \mathrm{~ms}$ SOA conditions in the change condition, $t(17)=$ $1.42,0.31, p \mathrm{~s}>0.17$, respectively (all tests are two-tailed).

\section{References}

Allen, R. J., Baddeley, A., \& Hitch, G. J. (2006). Is the binding of visual features in working memory resource-demanding? Journal of Experimental Psychology. General, 135, 298-313.

Baddeley, A. (1986). Working memory. Oxford: Oxford University Press.

Dell'Acqua, R., Sessa, P., Jolicoeur, P., \& Robitaille, N. (2006). Spatial attention freezes during the attentional blink. Psychophysiology, 43, 394-400.

Dell'Acqua, R., Sessa, P., Toffanin, P., Luria, R., \& Jolicoeur, P. (2010). Orienting attention to objects in visual short-term memory. Neuropsychologia, 48, 419-428.

Donkin, C., \& Nosofsky, R. M. (2012). The structure of short-term memory scanning: An investigation using response time distribution models. Psychonomic Bulletin \& Review, 19, 363-394.

Gazzaley, A., \& Nobre, A. C. (2012). Top-down modulation: Bridging selective attention and working memory. Trends in Cognitive Sciences, 16, 129-135.

Gilchrist, A. L., \& Cowan, N. (2014). A two-stage search of visual working memory: Investigating speed in the change-detection paradigm. Attention, Perception, \& Psychophysics, 76, 2013-2050.

Gratton, G. (1998). The contralateral organization of visual memory: A theoretical concept and a research tool. Psychophysiology, 35, 638647.

Green, C., Johnston, J. C., \& Ruthruff, E. (2011). Attentional limits in memory retrieval - Revisited. Journal of Experimental Psychology: Human Perception \& Performance, 37, 1083-1098.

Hyun, J. S., Woodman, G. F., Vogel, E. K., \& Hollingworth, A. (2009). The comparison of visual working memory representations with perceptual inputs. Journal of Experimental Psychology: Human Perception \& Performance, 35, 1140-1160.

Jolicoeur, P., \& Dell'Acqua, R. (1998). The demonstration of short-term consolidation. Cognitive Psychology, 36, 138-202.

Kuo, B.-C., Rao, A., Lepsien, J., \& Nobre, A. C. (2009). Searching for targets within the spatial layout of visual short-term memory. Journal of Neuroscience, 29, 8032-8038. 
Logan, G. D., \& Gordon, R. D. (2001). Executive control of visual attention in dual-task situations. Psychological Review, 108, 393-434.

Luck, S. J., \& Hillyard, S. A. (1994). Spatial filtering during visual search: Evidence from human electrophysiology. Journal of Experimental Psychology: Human Perception \& Performance, 20, $1000-1014$.

Luck, S. J., \& Vogel, E. K. (1997). The capacity of visual working memory for features and conjunctions. Nature, 390, 279-281.

Magen, H., \& Cohen, A. (2010). Modularity beyond perception: Evidence from the PRP paradigm. Journal of Experimental Psychology: Human Perception \& Performance, 36, 395-414.

Pashler, H. (1991). Shifting visual attention and selecting motor responses: Distinct attentional mechanisms. Journal of Experimental Psychology: Human Perception \& Performance, 17, 1023-1040.

Pashler, H. (1994). Dual task interference in simple tasks: Data and theory. Psychological Bulletin, 116, 220-244.

Phillips, W. A. (1974). On the distinction between sensory storage and shortterm visual memory. Perception \& Psychophysics, 16, 283-290.

Reimer, C. B., Strobach, T., Frensch, P. A., \& Schubert, T. (2015). Are processing limitations of visual attention and response selection subject to the same bottleneck in dual-tasks? Attention, Perception, \& Psychophysics, 77, 1052-1069.

Schweickert, R. (1980). Critical-path scheduling of mental processes in a dual task. Science, 209, 704-706.

Sternberg, S. (1966). High-speed scanning in human memory. Science, $153,652-654$.

Sternberg, S. (1975). Memory scanning: New findings and current controversies. Quarterly Journal of Experimental Psychology, 27, 1-32.

Theeuwes, J., Kramer, A. F., \& Irwin, D. E. (2011). Attention on our mind: The role of spatial attention in visual working memory. Acta Psychologica, 137, 248-251.

Townsend, J. T. (1990). Serial vs. parallel processing: Sometimes they look like Tweedledum and Tweedledee but they can (and should) be distinguished. Psychological Science, 1, 46-54.

Treisman, A., \& Zhang, W. (2006). Location and binding in visual working memory. Memory \& Cognition, 34, 1704-1719.

Yin, J., Gao, Z., Jin, X., Ding, X., Liang, J., \& Shen, M. (2012). The neural mechanisms of percept-memory comparison in visual working memory. Biological Psychology, 90, 71-79. 